\title{
ESDA2006-95218
}

\section{PhotoMEA: A NEW STEP TOWARDS TOTAL OPTICAL ANALISYS OF IN VITRO NEURONAL NETWORKS}

\author{
Diego Ghezzi
}

Politecnico di Milano Technical University, Bioengineering Department P.zza Leonardo da Vinci 32 - 20133 Milano

Tel: +39 0223999023, Fax: +39 0223999000, E-mail: diego.ghezzi@polimi.it

\section{Andrea Menegon}

San Raffaele Scientific Institute, DIBIT

Via Olgettina 60 - 20132 Milano

Tel: +39 0226434868, Fax: +39 0226434813, E-mail: menegon.andrea@hsr.it

\section{Alessandra Pedrocchi}

Politecnico di Milano Technical University, Bioengineering Department

P.zza Leonardo da Vinci 32 - 20133 Milano

Tel: +39 0223993346, Fax: +39 0223993360, E-mail: alessandra.pedrocchi@polimi.it

\section{Sara Mantero}

Politecnico di Milano Technical University, Bioengineering Department

P.zza Leonardo da Vinci 32 - 20133 Milano

Tel: +39 0223993346, Fax: +39 0223993360, E-mail: sara.mantero@polimi.it

\section{Flavia Valtorta}

San Raffaele "Vita-Salute" University

Via Olgettina 60 - 20132 Milano

Tel: +39 0226434826, Fax: +39 0226434813, E-mail: valtorta.flavia@hsr.it

\section{Giancarlo Ferrigno}

Politecnico di Milano Technical University, Bioengineering Department

P.zza Leonardo da Vinci 32 - 20133 Milano

Tel: +39 0223993371, Fax: +39 0223993360, E-mail: giancarlo.ferrigno@polimi.it 


\section{ABSTRACT}

Light stimulation of neurons is a promising approach for investigating the molecular mechanisms at the basis of neuronal physiology and plasticity. In particular, flash photolysis of caged compounds offers the unique advantage of allowing to quickly change the concentration of either intracellular or extracellular bioactive molecules, such as neurotransmitters or second messengers, for the stimulation or modulation of neuronal activity.

In this field of research, we describe a simple laser-based set-up for the local activation of caged compounds. The coupling of a UV laser diode to a small-core optical fibre allows to reduce the uncaging area and to quickly change the stimulation point. The actual localisation of the light stimulation is determined using a caged fluorescent compound (dextran, DMNB-caged fluorescein). The efficiency of our set up for neuronal stimulation is tested with a caged neurotransmitter (MNI-caged-L-glutamate). Activation of caged glutamate evokes neuronal responses that are recorded using a MicroElectrode Array system and/or following the variations in the concentrations of the $\mathrm{Ca}_{\mathrm{i}}{ }^{2+}$.

This work shows that our laser-based set-up is a powerful tool for local activation of caged compound allowing a unique opportunity to follow the effects of local neuronal pathways on neuronal network activity, for instance during pharmacological and toxicological treatments.

Keywords: Neuronal laser stimulation; Caged compound; Neuronal optical imaging; Uncaging by optical fibre;

\section{INTRODUCTION}

The most important function of neurons and neuronal networks is to process, modulate and transmit electro-chemical information. Our ability in eliciting, modulating and recording this activity is fundamental for deepening our knowledge of the mechanisms underlying the Central Nervous System (CNS) functions. In fact, higher brain functions originate both from the intrinsic capability of the neuron to modulate its activity, depending on its physiology as well as on its previous functional history, and from the additional capability to modulate the physiological state of the complex functional network.

In the last decade, studies on neuronal physiology and plasticity have provided a detailed picture of the molecular mechanisms underlying the modulation of neuronal activity; on the other hand, the molecular mechanisms which control the network properties remain poorly understood, and represent a new frontier in neuroscience.

Two different approaches can be followed for the study of neuronal functions: a large-scale approach aiming at understanding the activity of many neurons interacting in a complex network and a micro-scale approach aiming at providing detailed behavioural models of the molecular systems which actively contribute to the generation and modulation of the neuronal activity.

Higher brain functions are created by the synchronised activity of discrete regions involved in neuronal information processing, where a single neuron may influence the activity of many other neurons forming the neuronal functional network. Thus, the study of the modulation of neuronal physiology that regulates neuronal activity must necessarily be inserted into a large-scale view of the neuronal network dynamics.

A new breakthrough in neuroscience would be the possibility to stimulate and modulate a single neuron, or selected parts thereof, and study its influence over the functioning of the entire network. In this manner, the microscale meets the large-scale approach, allowing the understanding of how the mechanisms that influence the physiology of single neuronal units are able to alter the behaviour of the entire network.

At present, experiments on neuronal physiology and plasticity are typically carried out by electrical stimulations and recordings using intracellular or extracellular electrodes, as well as MicroElectrode Array devices (MEAs). These systems have yielded important results in electrophysiology and neurobiology but nowadays they are showing some limits. Electrophysiological studies of a single neuron can be traditionally performed by intracellular electrodes. This approach mechanically damages the cell and as a consequence alter the entire network. Moreover, intracellular electrodes do not allow studies of the whole neuronal network, but only of the single cells.

In contrast, extracellular electrodes, in particular MEAs [1-3], do not allow to perform local stimulations and highresolution recordings of the neuronal network, because of the unfocused electrical fields that spread in the medium. MEAs can be exploited for monitoring the electrical activity of the entire network, but with the limitation of recording only some discrete and ill-defined regions.

In addition to traditional electrophysiology techniques, optical methods for the local stimulation of neuronal activity have been used for relatively a long time. The ability to use light provides a tool for the precise spatial and temporal stimulation of different regions of a neuronal network, which can be sized down to sub-neuronal compartments.

In the last two decades, different methods for neuronal light stimulation have been proposed and reviewed [4]: direct laser stimulation [5], dye-mediated laser stimulation [6], direct twophoton excitation [7], endogenous expression of molecules sensitive to light [8] and caged neurotransmitters activation [9]. The use of caged compounds seems to be the most physiological approach for the coupling of light and neuronal excitation, e.g. with caged glutamate [10], or modulation, e.g. with caged intracellular second messengers [11]. In particular, since glutamate is the most important and widespread excitatory neurotransmitters in the brain, it seems the best solution to provide local and controlled stimulations to neurons or to any part of them.

The basic approach is to cage the compound with a chemical blocking group, typically a nitrobenzyl group, that can be removed by UV light pulses. Thus, the caged compound can be switched to the active form with a fine spatial and temporal control.

The typical set-up for the activation of caged compounds is based on an epifluorescence microscope, a UV light source, such as a xenon or mercury arc lamp coupled to an electromechanical shutter, or a flash lamp, for producing uncaging in the region of interest [12].

Currently, optical stimulation of neurons is still matched with electrical recordings of neuronal activity performed by intracellular electrodes or MEAs, with the consequence of the 
disadvantages linked to the electrical recordings.

Recently, a new device for neuronal cultures analysis, named PhotoMEA, has been proposed [13]. PhotoMEA will be a new biosensor based on innovative optical technologies that combines in a single chip the local light stimulation with high-spatial resolution fluorescent optical imaging over the entire culture.

The present work is the first step towards a complete optical analysis of cultured neuronal networks. A simple experimental set-up for the local optical stimulation and the optical recording of neuronal activity is shown and its validity is tested by classic MEA recordings.

Our long-term goal is the miniaturisation of the proposed set-up, providing a single chip for the complete optical analysis. This biosensor, based on optical microtechnologies, will provide the local stimulation with caged compounds and the recording from the whole network by fluorescent dyes, such as ion-sensitive or voltage-sensitive indicators.

In this direction, the merging of micro-scale and largescale approaches offers a unique opportunity to follow the effects of local neuronal pathways on neuronal network activity, for instance during pharmacological and toxicological treatments.

\section{MATERIALS AND METHODS Cell cultures}

Low-density primary cultures of hippocampal neurons were prepared from embryonic day 18 rat embryos (Charles River, Calco, Italy), essentially as previously described [14]. Hippocampi were dissociated by a $15 \mathrm{~min}$ incubation with $0.25 \%$ trypsin at $37^{\circ} \mathrm{C}$, and cells were plated at a density of $800 / \mathrm{mm}^{2}$ on poly-L-lysine $(1 \mathrm{mg} / \mathrm{ml}$ )-treated MEAs (Multi Channel Systems, Reutlingen, Germany) in MEM (Invitrogen, San Giuliano Milanese, Italy), supplemented with $10 \%$ horse serum (Hyclone, Logan, UT), 2mM glutamine (Biowhittaker, Ververs, Belgium), and 3.3mM glucose.

After allowing neurons to adhere to the surface for 3-4hr, a coverslip $(10 \mathrm{~mm})$ with a monolayer of cortical astrocytes [15] was turned over neurons. Physical contact between neurons and glial cells was avoided by placing paraffin dots in the MEA culture dish (Fig. 1).

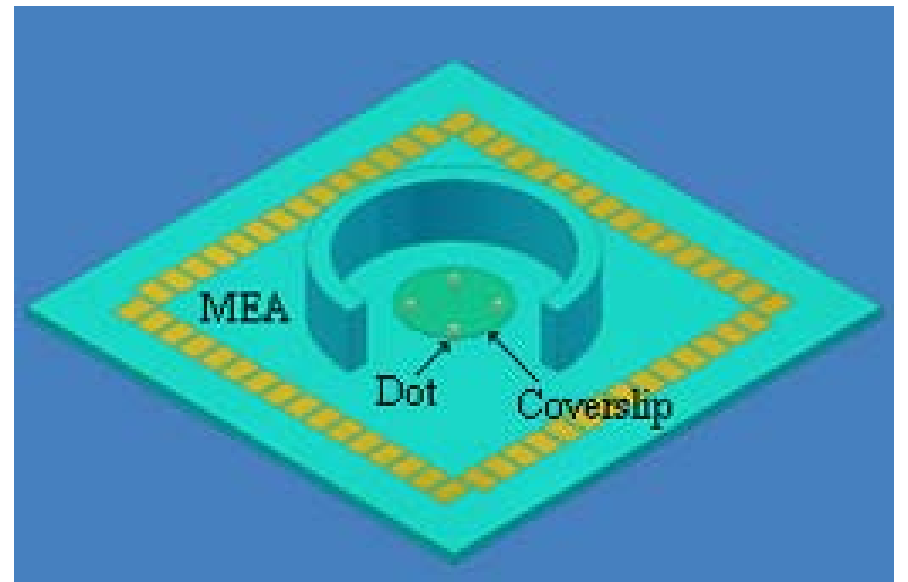

Fig. 1 - The rationale of our approach for culturing hippocampal neurons on MEAs: neurons and glial cells are combined to make a double layer, but they are separated by a narrow gap, so that the soluble substances can diffuse between them, without physical contact.
Cells were then kept in serum-free MEM supplemented with 1\% N2 supplement (Invitrogen, San Giuliano Milanese, Italy), $2 \mathrm{mM}$ glutamine (Biowhittaker, Ververs, Belgium), $0.1 \%$ ovalbumin (Sigma, St. Louis, MO), $4 \mathrm{mM}$ glucose, and $1 \mathrm{mM}$ sodium pyruvate. Evaporation of the medium was avoided using a membrane-sealed culture chamber [16].

Experiments were performed removing the coverslip and maintaining neurons in a Krebs-Ringer's solution buffered with HEPES (KRH; in mM: $150 \mathrm{NaCl}, 5 \mathrm{KCl}, 1.2 \mathrm{MgSO}_{4}$, $1.2 \mathrm{KH}_{2} \mathrm{PO}_{4}, 2 \mathrm{CaCl}_{2}, 10$ glucose, and $10 \mathrm{HEPES} / \mathrm{NAa}, \mathrm{pH}$ 7.4).

\section{Caged compounds}

MNI-caged-L-glutamate (4-Methoxy-7-nitroindolinylcaged L-glutamate, Tocris Bioscience, Bristol, UK) was dissolved in ethanol and, before the experiments, added to the experimental buffer solution (KRH), resulting in a $100 \mu \mathrm{M}$ concentration. This variant of caged glutamate is highly resistant to hydrolysis, its photolysis is fast (half time $\leq$ $0.26 \mathrm{~ms}$ ) [17] and the measured quantum yield is 0.085 at $347 \mathrm{~nm}[18]$.

Dextran, DMNB-caged fluorescenin (Invitrogen, San Giuliano Milanese, Italy), dissolved in PBS $(250 \mu \mathrm{g} / \mathrm{ml})$, was plated on poly-L-lysine $(1 \mathrm{mg} / \mathrm{ml})$-treated glass coverslip for $1 \mathrm{hr}$, and after, washed three times for $2 \mathrm{~min}$ in fresh PBS.

\section{Set-up description}

The set-up was built around an Axiovert 100 inverted epifluorescence microscope (Zeiss, Oberkochen, Germany) equipped with $32 \mathrm{x}$ and $20 \mathrm{x}$ long distance objective lenses, a mercury arc lamp and an ORCAII CCD camera (Hamamatsu, Shizuoka, Japan), positioned over an anti-vibration table.

Optical tools. A high power UV laser diode $(10 \mathrm{~mW} / 373 \mathrm{~nm})$ purchased from Nichia Corporation (NDHU110APAE2) was used as a light source. The laser diode was assembled into an OZ-2000 laser driver (OZ Optics, Ottawa, Canada) with a TTL external modulation (up to $3 \mathrm{kHz}$ ) and a temperature controller.

Laser output was focused into a single mode UV optical fibre (BC1197, $2 \mu \mathrm{m}$ core, $125 \mu \mathrm{m}$ clad, 0.12NA, OZ Optics, Ottawa, Canada) with high UV transmission. Coupling efficiency is $20 \%$, resulting in a $2 \mathrm{~mW}$ power output.

For the local uncaging, a special optical tool was built (Fig. 2). An optical fibre was glued in a glass ferule with the tip bulking out of the ferule above $5 \mathrm{~mm}$. The fibre was mounted on a computer assisted micromanipulator (Luigs \& Neumann GmbH, Ratingen, Germany) This allowed to place and move easily the optical tip in the interesting areas of the culture. Four different optical fibres from OZ Optics, with different core/clad ratio, were used: 2/125 (BC1197), 10/125 (BC1247), 25/125 (BC1251) and 50/125 (BC1259). Optical tips were joined to the laser diode by means of a standard $\mathrm{FC} / \mathrm{FC}$ connector.

The stimulation point can be easily changed during the experiment using the micromanipulator. To control the positioning a red laser diode $(5 \mathrm{~mW} / 633 \mathrm{~nm})$ was joined to the optical tool via the FC/FC connector. Substituting the UV laser with the red laser for testing the positioning, was useful also for avoiding any risk of uncaging during the preparatory positioning phase. 


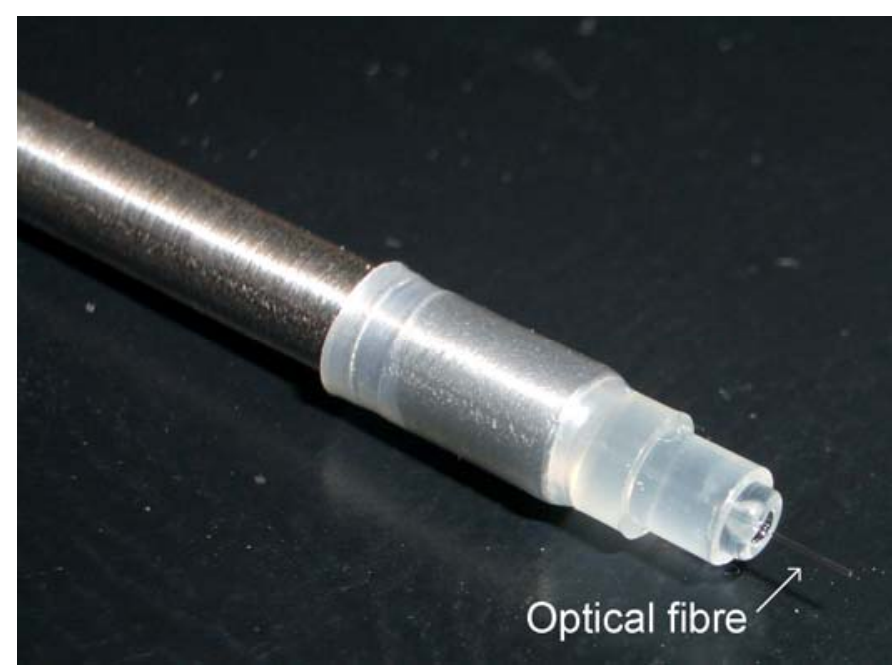

Fig. 2 - Optical tool for local neuronal stimulation.

Electrical recordings. Electrical recordings of the optically evoked neuronal activity was performed using the MEA chip equipped with a custom-built analog board for signal processing (Fig. 3). Neuronal responses were amplified (programmable gain ranging from 2,200x to $15,400 x$ ), lowpass filtered (programmable cut-off frequency ranging from $1,600 \mathrm{kHz}$ to $11,200 \mathrm{kHz}$ ) and sampled (sample rate $30 \mathrm{kHz}$ ) by a DAQ6024E data acquisition board (National Instruments, Austin, TX).

Experiments were performed at room temperature in $\mathrm{KRH}$ supplemented with MNI-caged glutamate $(100 \mu \mathrm{M})$.

Fluorescence imaging. $\mathrm{Ca}_{\mathrm{i}}^{2+}$ was measured using the $\mathrm{Ca}^{2+}$-sensitive fluorescent ratiometric indicator fura-2 (Invitrogen, San Giuliano Milanese, Italy).

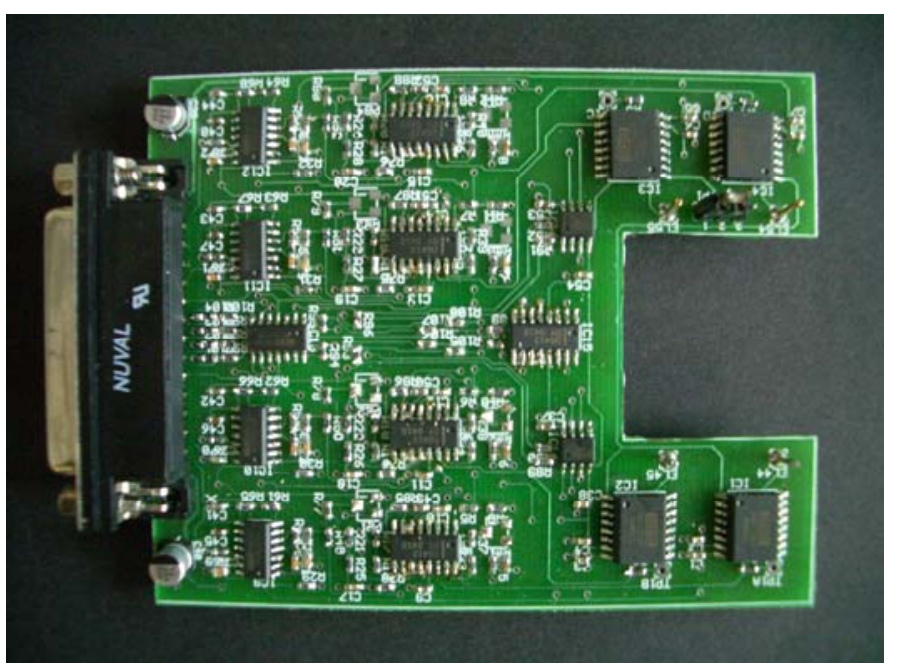

Fig. 3 - Analog board for signal processing.

Neurons were loaded for $1 \mathrm{hr}$ with $5 \mu \mathrm{M}$ fura-2AM in $\mathrm{KRH}$ at $37^{\circ} \mathrm{C}$, washed three times for $10 \mathrm{~min}$ in fresh $\mathrm{KRH}$ and transferred to the chamber of the microscope equipped with a $\mathrm{Ca}^{2+}$ imaging unit based on a modified CAM-230 dualwavelength microfluorometer (Jasco, Tokyo, Japan) as a light source. Experiments were performed in KRH supplemented with MNI-caged glutamate $(100 \mu \mathrm{M})$ at room temperature using the Axon Imaging Workbench 2.2 equipped with a Personal Computer Optics (Computer Optics GmbH, Kelheim, Germany) Super Video Graphics Array SensiCam (Axon Instruments, Foster City, CA). Images were acquired at 1-2 $340 / 380 \mathrm{ratios} / \mathrm{sec}$, and ratio values were calculated from sequences of images to obtain temporal analysis.

Figure 4 shows the scheme of the system for the optical analysis of neuronal activity combined with MEA recordings.

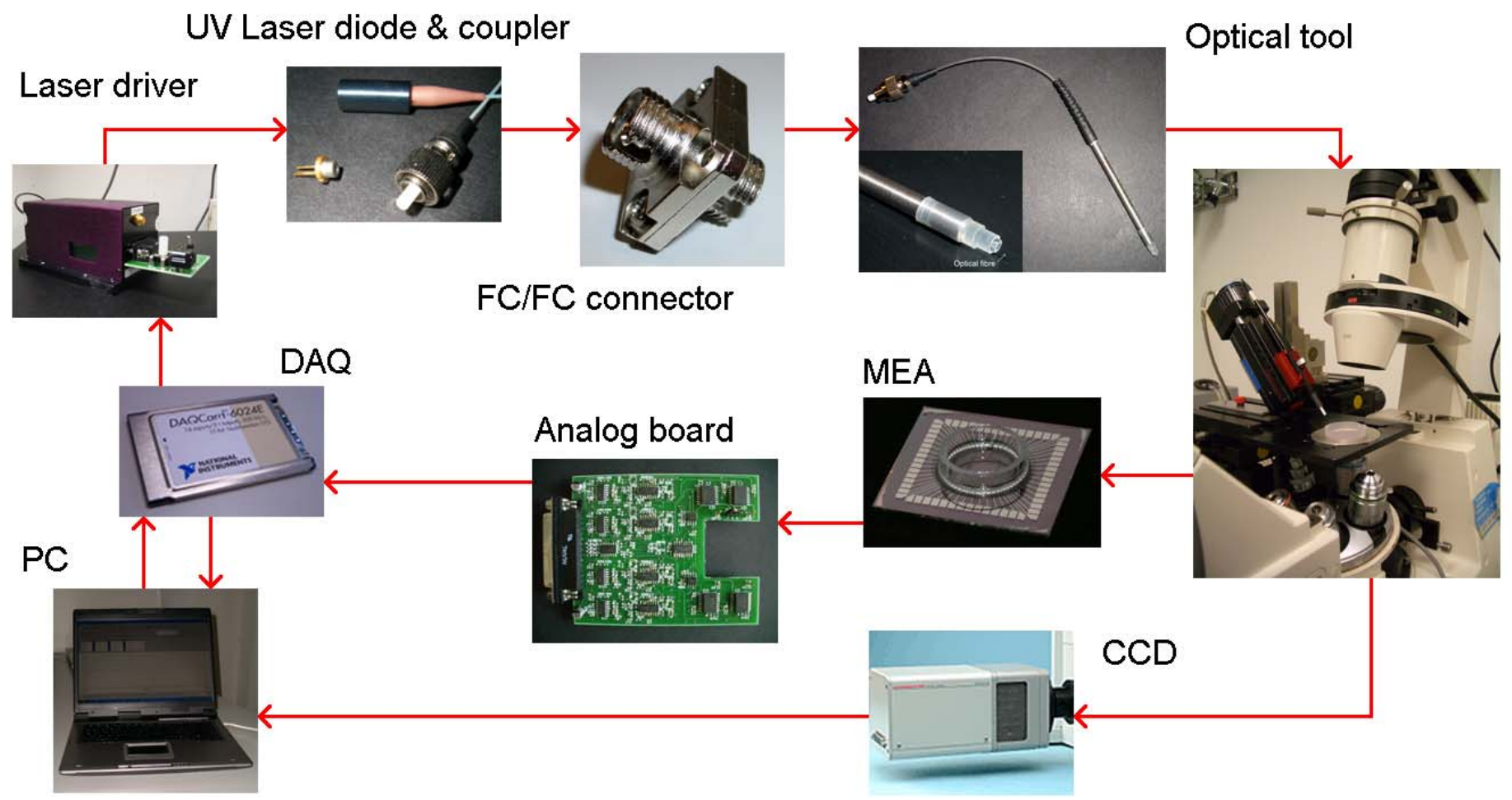

Fig. 4 - Scheme of the set-up for the local optical stimulation of neuronal networks combined with MEA and optical recordings of $\mathrm{Ca}^{2+}$. 


\section{RESULTS \\ Cell culture}

The method here described for low-density hippocampal neuronal cultures allows us to obtain glia-free cultures that survive for up to 20 days in vitro (DIV).

Our experiments were performed at 14DIV (Fig. 5), i.e. at this stage of development corresponding to mature cultures, characterised by a dense network of synaptic contacts.

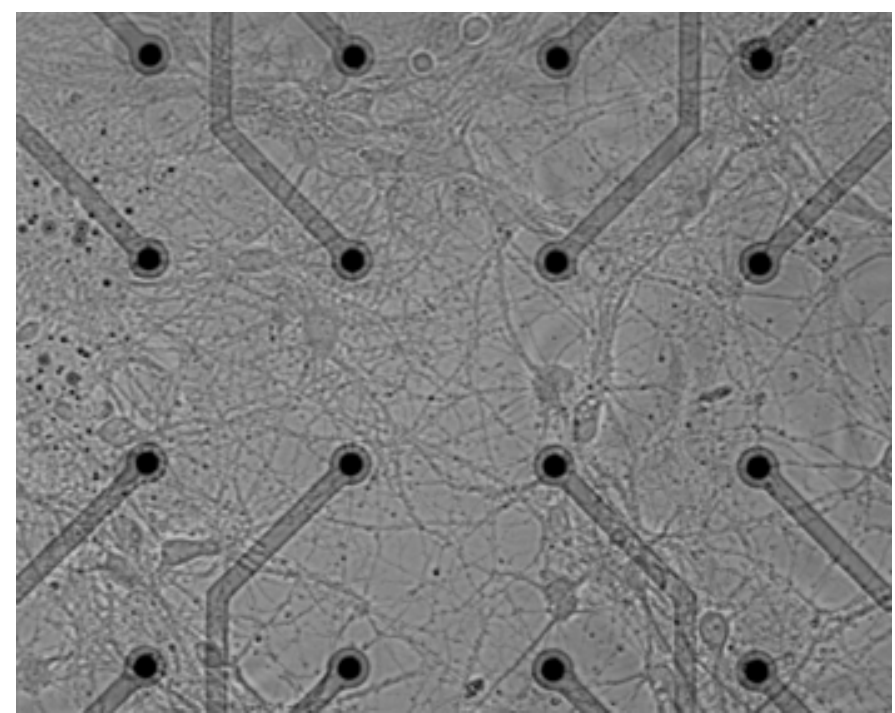

Fig. 5 - Low-density hippocampal neuronal cultures at 14DIV. The picture shows neurons around the sixteen central electrodes of the MEA chip.

\section{Local optical stimulation}

One of the most important feature of this laser-based setup is the capability to stimulate small regions of a neuronal network down to a part of the neuronal cell.

The capability of local stimulation was demonstrated in two steps. First, after placement of the fibre, we took a picture of the light spot obtained with the red laser diode.

Figure 6 shows the image of the light spot obtained with the $2 \mu \mathrm{m}$ optical fibre and acquired by the ORCAII CCD camera.

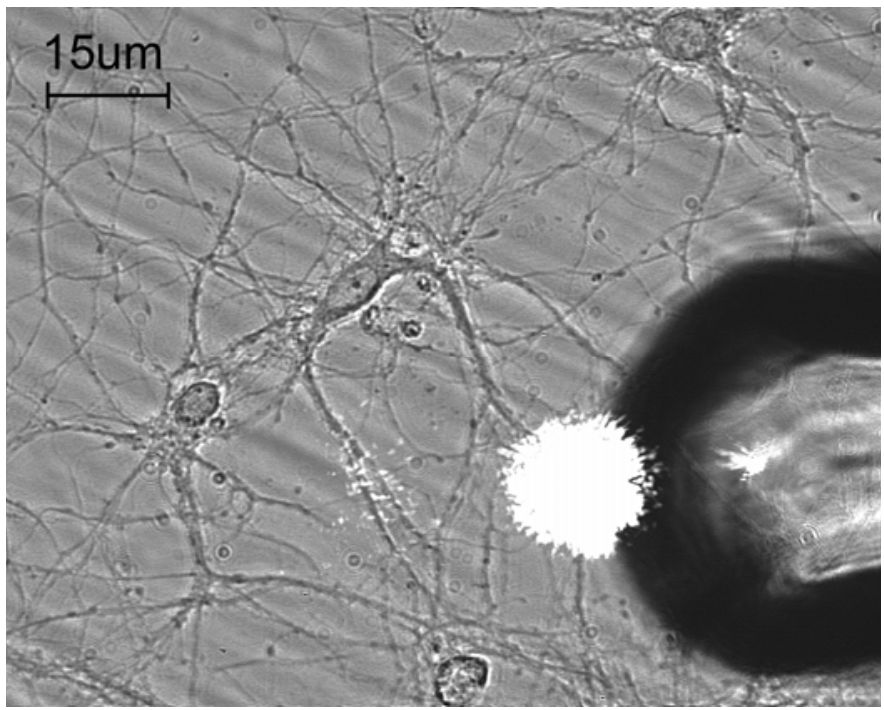

Fig. 6 - Light spot obtained by means of the red laser diode $(2 \mu \mathrm{m}$ fibre).
The size of the spot in the figure seems to be comparable to that of the cell body of the neuron. In this case a small portion of the dendritic tree of the neuron is stimulated. Thus, this picture shows that combining glia-free low density cultures of neurons with local optical stimulation allows us to stimulate the neuronal network at the sub-cellular level, and therefore to study at this level the mechanisms of plasticity in neuronal networks. Since the size of the light spot does not necessarily correspond to the actual area of photolysis, the uncaging area was also evaluated using a fluorescent caged compound (Dextran, DMNB-caged fluorescenin). Photolysis of caged dextran, attached to the surface of the glass coverslip, was achieved placing the $2 \mu \mathrm{m}$ optical fibre near the surface and illuminating the sample with a single pulse of $100 \mathrm{msec}$. The image (Fig. 7) of the fluorescent compound was acquired with the ORCAII CCD camera. Full-width-half-maximal intensity area has a size of $20 \mu \mathrm{m}$, comparable with the spot obtained by means the red laser .

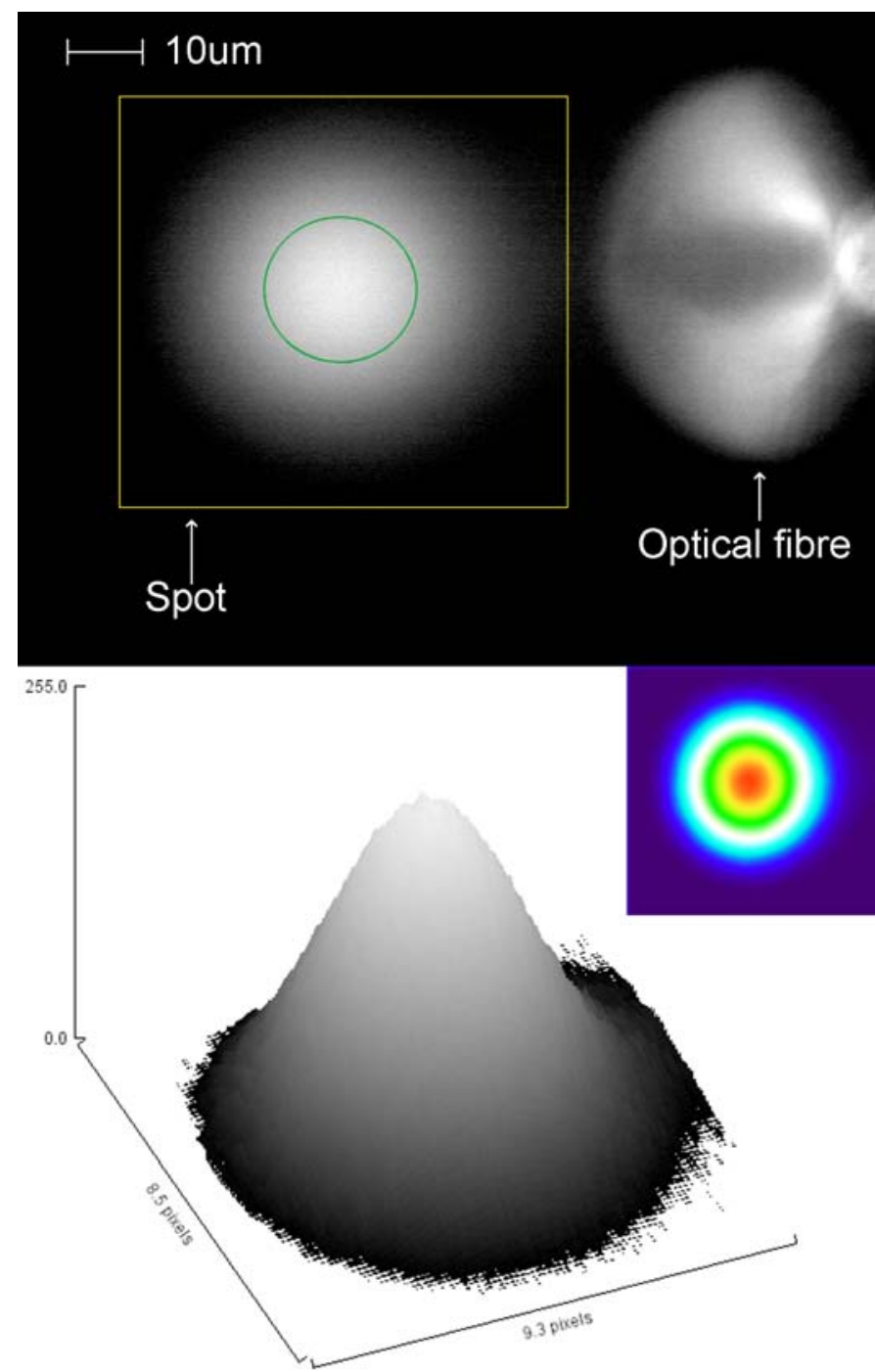

Fig. 7 - The top panel shows the fluorescence spot achieved with DMNB-caged fluorescenin following a $100 \mathrm{msec}$ light stimulus; the full-width-half-maximal (FWHM) intensity area is also represented. The bottom panel shows the three dimensional and pseudocolour representation of the intensity distribution. The uncaging area measured by DMND-caged fluorescenin seems to be comparable with the spot obtained with the red laser diode. 


\section{Electrical recordings}

In order to validate the optical stimulation using the caged compound, a test trial was done coupling optical stimulation with electrical recording by MEA.

MNI-caged-L-glutamate is a caged neurotransmitter used for the dendritic stimulation of neurons. The fibre for uncaging was placed closed to a neuron near to an electrode of the MEA; this electrode was used for recording neuronal responses. An optical stimulus of $100 \mathrm{msec}$ was delivered to the neurons and the response was recorded before and after the stimulation, by a classical MEA system (Fig. 8).

Another advantage of the optical stimulation is that do not present the stimulation artefact, as shown in the recorded trace.

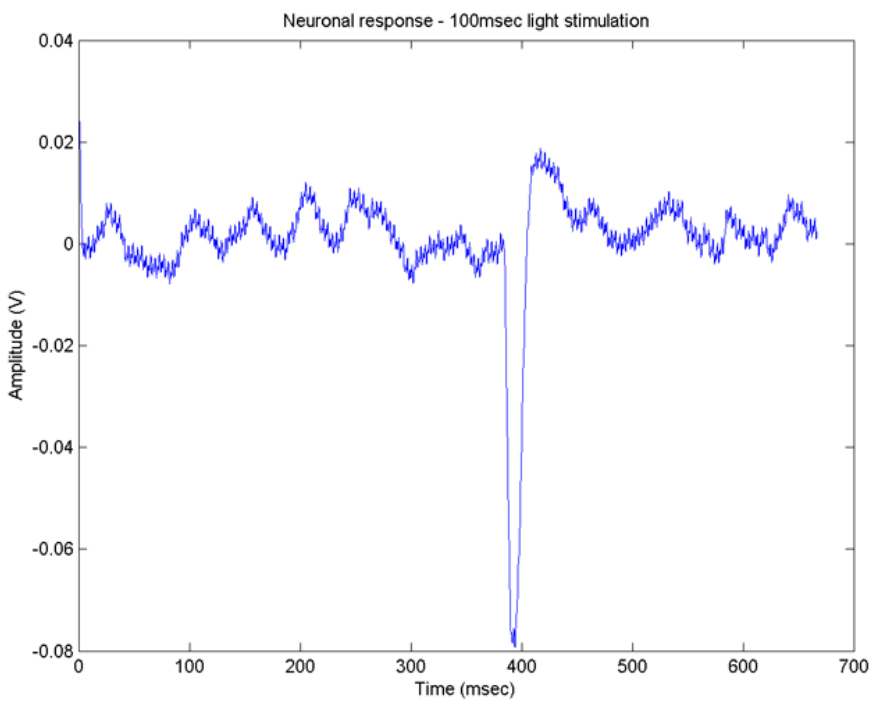

Fig. 8 - Neuronal electrical response evoked by a light pulse of $100 \mathrm{msec}$ in KRH supplemented with MNI-caged-L-glutamate, resulting in a $100 \mu \mathrm{M}$ concentration.

\section{Optical recordings}

Optical recording of the evoked neuronal activity was performed monitoring the variations of intracellular $\mathrm{Ca}^{2+}$ concentration by the $\mathrm{Ca}^{2+}$-sensitive fluorescent ratiometric indicator fura-2. Figure 9 shows the field where the stimulation was performed.

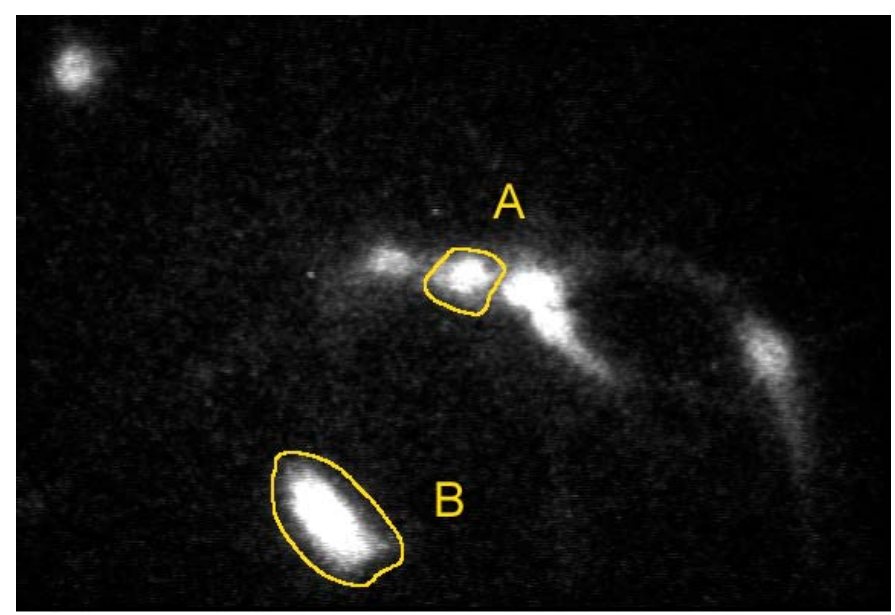

Fig. 9 - Image of the region of interest: two cells were stimulated with UV pulses in different trials: a neuronal cell (A) and a glial cell (B).
In order to obtain high-quality optical analysis, experiments with fura- 2 were performed positioning the optical fibre in a region without electrodes and conductive leads.

Pulses of different duration were delivered to the sample, showing that in this set-up pulses of $100 \mathrm{msec}$ are indicated for neuronal stimulation. Figure 10 shows the activation of the neuronal cell after the $100 \mathrm{msec}$ stimulus.

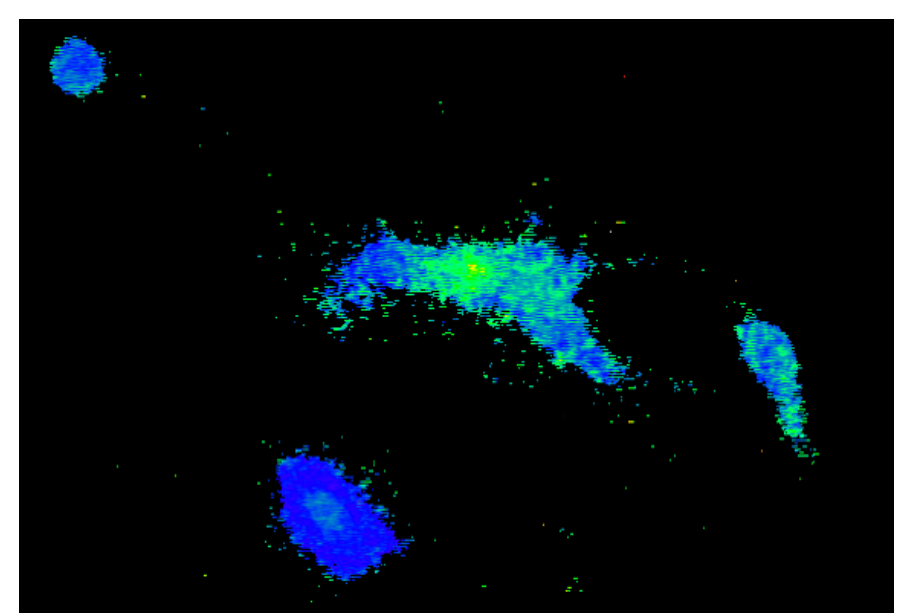

Fig. 10 - Activation of the neuronal cell after a 100msec UV pulse in $\mathrm{KRH}$ supplemented with MNI-caged-L-glutamate, resulting in a $100 \mu \mathrm{M}$ concentration.

Figure 11 shows the temporal analysis obtained both for neuronal cell and glial cell. The first period of $2 \mathrm{~min}$ shows that the intensity of light for fluorescent excitation of fura-2 $(340 / 380 \mathrm{~nm})$ is too low for activating caged glutamate.

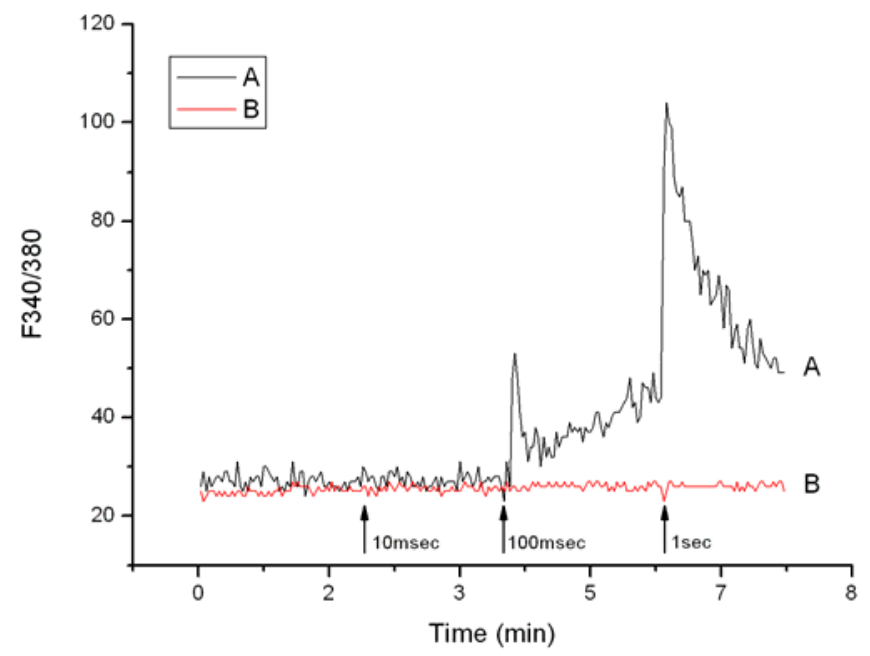

Fig. 11 - Three consecutives UV flashes of $10 \mathrm{msec}, 100 \mathrm{msec}$ and $1 \mathrm{sec}$ (black arrows) were delivered thorough the $2 \mu \mathrm{m}$ optical fibre towards both the neuronal cell (black trace A) and the glial cell (red trace B). The glial cell does not respond to any stimulus. The neuron does not respond to the first stimulus $(10 \mathrm{msec})$, responds to the second $(100 \mathrm{msec})$ with a quick recovery and presents a large response at the third stimulus, with recovery slow and not complete even after 1 minute.

Figure 11 also shows that the elevation in $\mathrm{Ca}^{2+}$ recorded in the experiment was the effect of the optical stimulation and not a pure artefact of such stimulus. 
This appears from the tracking activity of a glial cell close to the neuron where no change was recorded (red trace in Fig. 11). This experiment shows that the optimal pulse duration for this set-up is about $100 \mathrm{msec}$. Brief light pulses $(10 \mathrm{msec})$ could be unable to stimulate neuronal cells and too long pulses $(1 \mathrm{sec})$ could produce a saturated response of the neuron that could be not easy to recover.

\section{CONCLUSIONS}

Photorelease of caged compounds, e.g. light stimulation of neurons with caged glutamate, is a powerful tool for studying the molecular mechanisms at the basis of neuronal plasticity. In this study we present the use of a laser-based setup for the local activation of caged compounds.

Traditionally, different methodological solutions were adopted for optical stimulation by caged compounds. Local activation of caged compounds was performed using the objective lens of an epifluorescence microscope coupled with a high power UV source, such as the fluorescence lamp, with an electro-mechanical shutter.

Alternatively, flash lamps were used, but unfortunately they produced very large electromagnetic artefacts and required a considerable amount of time to be re-charged. The third possible solution tested was based on the use of confocal microscopes, allowing very precise local stimulation with the well known breakthrough of the costs.

This study shows the advantages achieved using a lowcost laser diode combined with a small-core optical fibre for local stimulation and modulation of neuronal networks. We show that the laser diode provides enough energy to uncage the compound tested (MNI-caged-L-glutamate) and the focal point of the uncaging can be sized down to selected parts of the neuron, such as its dendritic tree.

Using an optical fibre for the local uncaging, stimulation can be performed in any part of the culture. For instance, the activation of the caged compound can be performed away from the field of view of the microscope, allowing the possibility to couple the light stimulation with optical imaging. It has to be underlined that the proposed setup can be coupled with different microscopes for optical recordings, opening the chance to couple the optical stimulation with many wellknown fluorescence based methodologies.

This novel set-up opens exciting perspectives in many fields of neurobiology and neuroelectrophysiology. Thus, it allows analyzing in great details the activity of the neurotransmitter glutamate in the stimulation of selected neuronal compartments and revealing the extent of spreading of a localized signal by optical imaging.

In addition, the possibility of studying the relative contribution that an identified neuron has in the economy of an entire neuronal network may help in elucidating whether different sub-regions or neuronal subtypes play distinct roles in the flux of electrical information flowing within the meshwork.

Finally, a large-scale view of neuronal intercommunications is of great interest in the elucidation of the mechanisms of action of neurotropic drugs and offers a unique opportunity for testing their ability to affect neuronal properties as well as alterations in the behaviour of the entire network.

\section{FUTURE DEVELOMPENTS}

This set-up represents the first attempt towards the use of light for both neuronal stimulation and recording. At present, the set-up is built around an inverted epifluorescence microscope allowing virtually any optical analysis of the neuronal activity.

One of the purposes of our work is the development of a simple and low-cost set-up for providing a complete characterisation of the caged compound behaviour, in terms of concentrations of the compound, power of the light stimulus, dimension of the region of interest and duration of the pulse.

The final goal is the reproduction of the set-up in a miniaturised form, e.g. a single chip that allows both stimulations and recordings in a totally optical way. Nowadays, optical technologies provide methods for integrating the basic concepts of the light stimulation and optical recording in a single chip, similar to MEAs but with the advantages provided by the optical analysis. The main advantages of this chip, the PhotoMEA biosensor, are (1) the possibility to stimulate a neuron or selected parts thereof in a physiological manner using caged neurotransmitters, (2) the possibility to perform optical recordings of the neuronal activity with quite high-resolution using VSDs or other fluorescent dyes and (3) the possibility to avoid the use of a microscope performing optical imaging, hence simplifying the experimental set-up and eventually performing long-term analysis in an incubator or in a bioreactor.

\section{ACKNOWLEDGEMENTS}

This work was carried out in collaboration with the ALEMBIC facility (Advanced Light and Electron Microscopy BioImaging Center) of the San Raffaele Scientific Institute. A special thank goes to Fabio Grohovaz, director of the ALEMBIC facility. The authors wish to thank all the people that helped us to develop the set-up proposed. A special thank goes to Daniel Wagenaar (UCSD, La Jolla, CA) who gave us a valuable help to the development of the electrical part of the system and the software for data processing. Finally we wish to express our gratitude to Laura Monti for her substantial contribute to this work.

\section{REFERENCES}

[1] Thomas, C. A., Springer, P. A., Loeb, G. E., BerwaldNetter, Y., and Okun, L. M., 1971, "A miniature microelectrode array to monitor the bioelectric activity of cultured cells," Exp. Cell. Res., 74(1), pp. 61-66.

[2] Gross, G. W., 1979, "Simultaneous single unit recording in vitro with photoetched laser deinsulated gold multimicroelectrode surface," IEEE Trans. Biomed. Eng., 26(5), pp. 273-279.

[3] Pine, J., 1980, "Recording action potentials from cultured neurons with extracellular microcircuit electrodes," J. Neurosci. Methods, 2(1), pp. 19-31.

[4] Callaway, E. M., and Yuste, R., 2002, "Stimulating neurons with light," Curr. Opin. Neurobiol., 12(5), pp. 587592.

[5] Fork, R. L., 1971, "Laser stimulation of nerve cells in Aplysia," Science, 171(974), pp. 907-908.

[6] Farber, I. C., and Grinvald, A., 1983, "Identification of presynaptic neurons by laser photostimulation," Science, 
222(4627), pp. 1025-1027.

[7] Hirase, H., Nikolenko, V., and Goldberg, J. H., 2002, "Multiphoton stimulation of neurons," J. Neurobiol., 51(3), pp. 237-247.

[8] Zemelman, B. V., Lee, G. A., Ng, M., and Miesenbock, G., 2002, "Selective photostimulation of genetically charged neurons," Neuron, 33(1), pp. 15-22.

[9] Callaway, E. M., and Katz, L. C., 1993, "Photostimulation using caged glutamate reveals functional circuitry in living brain slices,” Proc. Natl. Acad. Sci. USA, 90(16), 7661-7665.

[10] Wieboldt, R., Gee, K. R., Niu, L., Ramesh D., Carpenter B. K., and Hess, G. P., 1994, "Photolabile precursors of glutamate: synthesis, photochemical properties, and activation of glutamate receptors on a microsecond time scale," Proc. Natl. Acad. Sci. USA, 91(19), pp. 8752-8756.

[11] Nerbonne, J. M., 1996, "Caged compounds: tools for illuminatine neuronal responses and connections," Curr. Opin. Neurobiol., 6(3), pp. 379-386.

[12] Kötter, R., Schubert, D., Dyhrfjeld-Johnsen, J., Luhmann, H. J., and Staiger, J. F., 2005, "Optical release of caged glutamate for stimulation neurons in the in vitro slice preparation,” J. Biomed. Opt., 10(1), 11003.
[13] Patent pending number MI2005A000114, assigned to Politecnico di Milano, Technical University.

[14] Banker, G. A., and Cowman, W. M., 1977, "Rat hippocampal neurons in dispersed cell culture," Brain Res., 126(3), pp. 397-342.

[15] Booher, J., and Sensenbrenner, M., 1972, "Growth and cultivation of dissociated neurons and glial cells from embryonic chick, rat and human brain in flask cultures," Neurobioloy, 2(3), pp. 97-105.

[16] Potter, S. M., and DeMarse, T. B., 2001, "A new approach to neural cell culture for long-term studies," J. Neurosci. Methods, 110(1-2), pp. 17-24.

[17] Canepari, M., Nelson, L., Papageorgiou, G., Corrie, J. E., and Ogden, D., 2001, "Photochemical and pharmacological evaluation of 7-nitroindolinyl-and 4-methoxy-7-nitroindolinylamino acids as novel, fast caged neurotransmitters," J. Neurosci. Methods, 112(1), pp. 29-42.

[18] Papageorgiou, G., and Corrie, J. E. T., 2000, "Effects of aromatic substituents on the photocleavage of 1-acyl-7nitroindolines," Tetrahedron, 56(41), pp. 8197-8205. 